21st Particles and Nuclei International Conference (PANIC 2017)

International Journal of Modern Physics: Conference Series

Vol. 46 (2018) 1860062 (6 pages)

(C) The Author(s)

DOI: $10.1142 / \mathrm{S} 2010194518600625$

\title{
Charm Physics Prospects at the Belle II Experiment
}

\author{
Long-Ke Li (on behalf of the Belle II Collaboration) \\ Institute of High Energy Physics, CAS \\ 19B Yuquan Road, Beijing City, 100049, China \\ lilongke@ihep.ac.cn
}

Published 3 May 2018

\begin{abstract}
With a total integrated luminosity of $50 \mathrm{ab}^{-1}$ of data and improved performances at the Belle II detector, especially vertex resolution and particle identification, sensitivity estimations for $D^{0}-\bar{D}^{0}$ mixing, $C P$ violation and time integrated $C P$ asymmetries measurements are presented. Prospects on charm rare decays and (semi-)leptonic decays are discussed. Besides, a new $D^{0}$ flavor-tagging technique, ROE method, is introduced.
\end{abstract}

Keywords: $D^{0}-\bar{D}^{0}$ mixing; $C P$ violation; rare and leptonic decay; ROE method; Belle II.

\section{SuperKEKB and Belle II}

As next generation of B factory, SuperKEKB, is upgraded from KEKB with the so-called Nano-Beam scheme, of which the basic idea is to squeeze the vertical beta function $\beta_{y}^{*}$ at interaction point(IP) by minimizing the longitudinal size of overlap region of the two beams at IP. Comparing to KEKB, SuperKEKB will improve the beta function and beam current a factor of twenty and two respectively, and it is designed to achieve a peak luminosity, $\mathcal{L}=8.0 \times 10^{35} \mathrm{~cm}^{-2} \mathrm{~s}^{-1}$, a factor of forty times improvement.

The Belle II detector is expected to have great performances in the reconstruction of final states with neutral particles (e.g. $\gamma, \pi^{0}, \eta$ ) and missing energy. It is mostly installed and commissioned, and schedules to collect the first data in the early of 2018 without vertex detector, and then to target a total integrated luminosity of $50 \mathrm{ab}^{-1}$ within next decade. Therefore Belle II will achieve an abundant $c \bar{c}$ events data sample, which provides a rich charm physics program to improve the precision of $D^{0}-\bar{D}^{0}$ mixing, time-dependent $C P$ violation, time-integrated $C P$ asymmetries and rare and (semi-)leptonic decay measurements.

\section{2. $D^{0}$ proper time resolution}

In Belle II detector, there will be a six-layer silicon vertex detector (two layers of pixel detectors and four layers of double-sided silicon strip detectors). And the

This is an Open Access article published by World Scientific Publishing Company. It is distributed under the terms of the Creative Commons Attribution 4.0 (CC-BY) License. Further distribution of this work is permitted, provided the original work is properly cited. 
innermost layer will be twice closer to the interaction point and the outmost layer will be larger than Belle's ${ }^{1}$. This will contribute to that $D^{0}$ decay vertex reconstruction with a improvement of efficiency comparing with Belle and BaBar. The vertex resolution will also be improved significantly. Time-dependent measurements will benefit from these improvements, in particular the precise of the determination of $D^{0}$ proper time, which is essential in time-dependent measurements.

According to Monte Carlo (MC) simulations, Belle II will have a $D^{0}$ proper time resolution $140 \mathrm{fs},{ }^{2}$, which is a factor of two improvement over Belle and BaBar (270 fs). The time resolution $\sigma_{t}$ with a factor of three improvement, and the root mean square of it is reduced by a factor of two, which will results in an increased statistics because of the fact that the width of resolution function of lifetime usually is related on $\sigma_{t}$ at B factories, such as $\operatorname{Res}(t)=\operatorname{Gauss}\left(\mu, k \sigma_{t}\right)$.

\section{3. $D^{0}-\bar{D}^{0}$ Mixing and $C P$ Violation}

One emphasis of the Belle II charm physics program is to make high precision measurements of $D^{0}-\bar{D}^{0}$ mixing parameters $x=\Delta M / \Gamma$ and $y=\Delta \Gamma / 2 \Gamma$, and of $C P$ violating parameters $|q / p|$ and $\arg (q / p)=\phi$. These measurements typically depend on measuring the decay time of $D^{0}$ mesons.

For sensitivity estimation in $D^{0} \rightarrow K^{+} \pi^{-}$decay, an ensemble of a thousand Toy-MC experiments is generated with separate samples of $D^{*+} \rightarrow D^{0} \pi_{s}^{+}, D^{0} \rightarrow$ $K^{+} \pi^{-}$and conjugated decays corresponding to $5 \mathrm{ab}^{-1}, 20 \mathrm{ab}^{-1}$, and $50 \mathrm{ab}^{-1}$ of data. The probability density function of decay rates in wrong-sign(WS), which is contributed by two processes: a double Cabbibo suppressed(DCS) process and a Cabbibo favored $(\mathrm{CF})$ process following mixing process, with $D^{0}-\bar{D}^{0}$ mixing and CPV-allowed is calculated by Eqn (1).

$$
\frac{d N_{D^{0} \rightarrow f}}{d t}=e^{-\Gamma t}\left[R_{D}+\left|\frac{q}{p}\right| \sqrt{R_{D}}\left(y^{\prime} \cos \phi-x^{\prime} \sin \phi\right) \Gamma t+\left|\frac{q}{p}\right|^{2} \frac{x^{\prime 2}+y^{\prime 2}}{4}(\Gamma t)^{2}\right](1)
$$

where effective mixing parameters $x^{\prime}=x \cos \delta+y \sin \delta$ and $y^{\prime}=y \cos \delta-x \sin \delta$ with strong phase difference $\delta$ between $D^{0} \rightarrow K^{+} \pi^{-}$and $\bar{D}^{0} \rightarrow K^{-} \pi^{+}$. These decay times are smeared with time resolution using a Gaussian with a width $\sigma=140 \mathrm{fs}$. Then $D$ lifetime distributions are fitted with mixing parameters $x^{\prime}, y^{\prime}$ in the case of no $C P$ violation, or together with $|q / p|$ and $\phi$ allowing for CPV. The previous Belle measurements and the estimations of expected sensitivity of $D^{0}-\bar{D}^{0}$ mixing and $C P$ violation parameters with several luminosities are listed in Table 1.

The sensitivity estimation of $D^{0}-\bar{D}^{0}$ mixing parameters $x^{\prime \prime}$ and $y^{\prime \prime}$ in WS $D^{0} \rightarrow$ $K^{+} \pi^{-} \pi^{0}$ decay is performed with Toy-MC simulation studies, by generating ten independent data sets with 225 thousands WS events for each ${ }^{3}$, which has assumed a similar efficiency with BaBar. Considering $|x|,|y| \ll 1$ and $C P$ conservation $(q / p=$ $1)$, the decay rate of WS decay $D^{0} \rightarrow \bar{f}$ is defined as:

$$
\left|\mathcal{M}_{\bar{f}}(t)\right|^{2}=e^{-\Gamma t}\left\{r_{0}^{2}\left|\mathcal{A}_{\bar{f}}^{D C S}\right|^{2}-r_{0}\left(A y^{\prime \prime}+B x^{\prime \prime}\right) \Gamma t+\frac{x^{\prime \prime 2}+y^{\prime \prime 2}}{4}\left|\overline{\mathcal{A}}_{\bar{f}}^{C F}\right|^{2}(\Gamma t)^{2}\right\}
$$


Table 1. The sensitivity estimation of $D^{0}-\bar{D}^{0}$ mixing parameters $x^{\prime}, y^{\prime}$ and $C P$ violation parameters $|q / p|$, $\phi$ of flavor tagged $D^{0} \rightarrow K^{+} \pi^{-}$decays at Belle II, under the condition of CPV-absence or CPV-allowed.

\begin{tabular}{|c|c|c|c|c|c|}
\hline \multirow[t]{2}{*}{ Condition } & \multirow{2}{*}{ Parameters } & \multirow{2}{*}{$\begin{array}{l}\text { Belle measurement } \\
\qquad 976 \mathrm{fb}^{-1}\end{array}$} & \multicolumn{3}{|c|}{ Belle II estimation } \\
\hline & & & $5 \mathrm{ab}^{-1}$ & $20 a b^{-1}$ & $50 \mathrm{ab}^{-1}$ \\
\hline no & $\sigma\left(x^{\prime 2}\right)\left(10^{-5}\right)$ & 22 & 7.5 & 3.7 & 2.3 \\
\hline $\mathrm{CPV}$ & $\sigma\left(y^{\prime}\right)(\%)$ & 0.34 & 0.11 & 0.056 & 0.035 \\
\hline \multirow{4}{*}{$\begin{array}{l}\text { CPV- } \\
\text { allowed }\end{array}$} & $\sigma\left(x^{\prime}\right)(\%)$ & & 0.37 & 0.23 & 0.15 \\
\hline & $\sigma\left(y^{\prime}\right)(\%)$ & & 0.26 & 0.17 & 0.10 \\
\hline & $\sigma(|q / p|)$ & & 0.197 & 0.089 & 0.051 \\
\hline & $\sigma(\phi)\left(^{\circ}\right)$ & & 15.5 & 9.2 & 5.7 \\
\hline
\end{tabular}

where $A=\Re\left(\mathcal{A}_{\bar{f}}^{D C S} \overline{\mathcal{A}}^{*}{ }_{\bar{f}}^{C F}\right), B=\Im\left(\mathcal{A}_{\bar{f}}^{D C S} \overline{\mathcal{A}}^{*}{ }_{\bar{f}}^{C F}\right), r_{0}$ is the modulus of the relative complex number between CF and DCS amplitudes, and $\mathcal{A}_{\bar{f}}^{D C S}$ and $\overline{\mathcal{A}}_{\bar{f}}^{C F}$ are normalized shapes on the Dalitz plot region. The effective mixing parameters $x^{\prime \prime}$ and $y^{\prime \prime}$ could be extracted by performing a time-dependent Dalitz plot fitting of the WS sample.

The mixing parameters $\left(x, y, \delta, 1 / r_{0}\right)=\left(2.58 \%, 0.39 \%, 10^{\circ}, 13.8\right)$ in Eqn $(2)$ are fixed as WS generator input parameters. The $D^{0}$ lifetimes of generated MC samples are smeared with a time resolution with $\sigma=140 \mathrm{fs}$. We fit the samples for $D^{0}-\bar{D}^{0}$ mixing parameters as well as the magnitudes and phases of the intermediate states. One of the time-dependent Dalitz plot fittings is illustrated in Fig. 1. From the fitting results of ten experiments, the sensitivity estimation are obtained as $\sigma_{x}=$ $0.057 \%$ and $\sigma_{y}=0.049 \%$ without including systematic uncertainties and the effect of backgrounds. By scaling the presence of backgrounds of approximately less than $40 \%$ on $x$ and $y$ by estimation on Belle results, we obtain the uncertainties of $\sigma_{x}=0.080 \%$ and $\sigma_{y}=0.070 \%$, which are almost an order of magnitude improvement.

\section{Time-integrated $C P$ asymmetries}

As introduced above, Belle II will have excellent efficiency for reconstructing multibody final states with very small detector-based asymmetries. Thus she is ideal for searching for time-integrated $C P$ asymmetries in a variety of final states. The sensitivity estimations at Belle II by scaling the Belle results with its statistics in Eqn(3) are shown in Table 2, where the results from Belle measurements are also list as comparison.

$$
\sigma_{\text {Belle II }}=\sqrt{\left(\sigma_{\text {stat }}^{2}+\sigma_{\text {syst }}^{2}\right) \cdot\left(\mathcal{L}_{\text {Belle }} / 50 \mathrm{ab}^{-1}\right)+\sigma_{\text {irred }}^{2}}
$$

In particular, $D^{0} \rightarrow K_{S}^{0} K_{S}^{0}$, a promising golden channel amongst the single Cabbibo suppressed decays for $C P$ violation measurement, which could be enhanced to an observable level in the Standard $\operatorname{Model}(\mathrm{SM})^{4}$, where the uncertainty is expected to be $\sigma=0.20 \%$ at Belle II. For the decay $D^{+} \rightarrow \pi^{0} \pi^{+}$, the $C P$ violation is zero as 

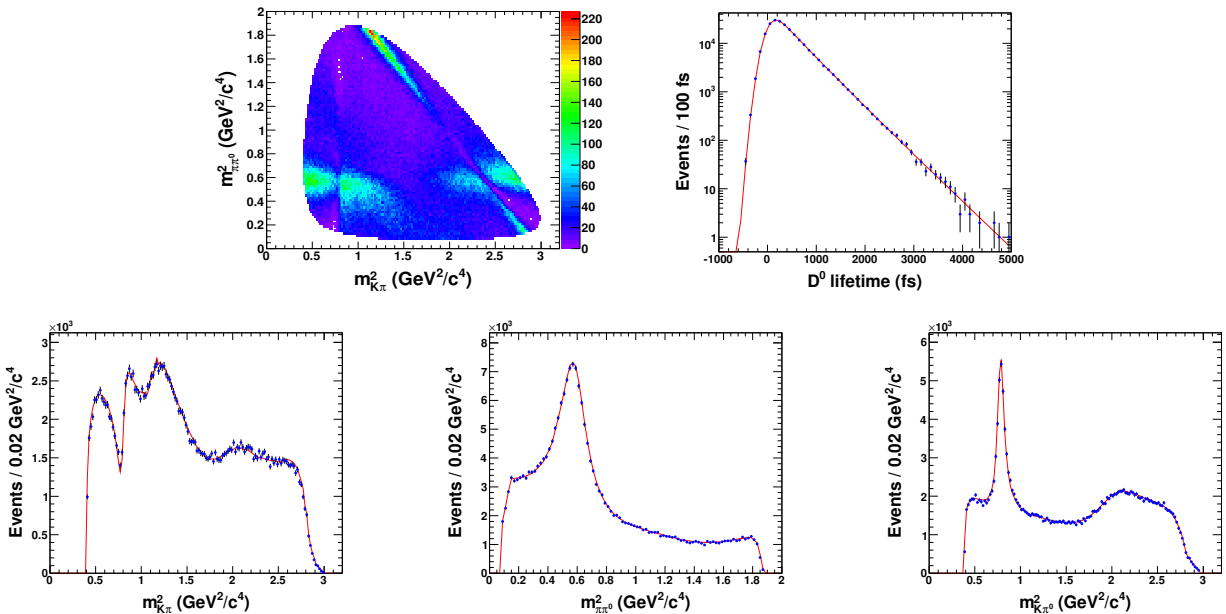

Fig. 1. Time-dependent Dalitz plot fit to the lifetime-smeared sample of wrong-sign $D^{0} \rightarrow$ $K^{+} \pi^{-} \pi^{0}$ decays. The decay times are smeared by the expected Belle II decay-time resolution of $140 \mathrm{fs}$. The second row shows projections of the fitted Dalitz variables $m_{K^{+} \pi^{-}}^{2}($ left $), m_{\pi^{-}}^{2}{ }_{\pi^{0}}$ (middle), and $m_{K^{+} \pi^{0}}^{2}$ (right).

Table 2. Time-integrated $C P$ asymmetries measurements at Belle experiment, and their corresponding uncertainties estimated at Belle II experiment with $50 \mathrm{ab}^{-1}$ of dataset and $\mathrm{LHCb}$ experiment with $50 \mathrm{fb}^{-1}$ of dataset.

\begin{tabular}{|c|c|c|c|c|c|}
\hline \multirow[t]{2}{*}{ Channel } & \multicolumn{3}{|c|}{ Current measurement } & \multicolumn{2}{|c|}{ Belle II LHCb } \\
\hline & $\mathcal{L}(/ \mathrm{fb})$ & $A_{C P}(\%)$ & References & $\sigma(\%)$ & $\sigma(\%)$ \\
\hline$D^{0} \rightarrow \pi^{+} \pi^{-}$ & 976 & $+0.55 \pm 0.36 \pm 0.09$ & PoS ICHEP2012 (2013) 353 & \pm 0.05 & \pm 0.03 \\
\hline$D^{0} \rightarrow K^{+} K^{-}$ & 976 & $-0.32 \pm 0.21 \pm 0.09$ & PoS ICHEP2012 (2013) 353 & \pm 0.03 & \pm 0.03 \\
\hline$D^{0} \rightarrow \pi^{0} \pi^{0}$ & 966 & $-0.03 \pm 0.64 \pm 0.10$ & PRL 112, 211601 (2014) & \pm 0.09 & \\
\hline$D^{0} \rightarrow K_{S}^{0} K_{S}^{0}$ & 921 & $-0.02 \pm 1.53 \pm 0.17$ & PRL 119, 171801 (2017) & \pm 0.20 & \\
\hline$D^{0} \rightarrow K_{S}^{0} \pi^{0}$ & 966 & $-0.21 \pm 0.16 \pm 0.07$ & PRL 112, 211601 (2014) & \pm 0.03 & \\
\hline$D^{0} \rightarrow \stackrel{\mathrm{K}}{K_{S}^{0}} \eta$ & 791 & $+0.54 \pm 0.51 \pm 0.16$ & PRL 106, 211801 (2011) & \pm 0.07 & \\
\hline$D^{0} \rightarrow K_{S}^{0} \eta^{\prime}$ & 791 & $+0.98 \pm 0.67 \pm 0.14$ & PRL 106, 211801 (2011) & \pm 0.09 & \\
\hline$D^{0} \rightarrow \pi^{+} \pi^{-} \pi^{0}$ & 532 & $+0.43 \pm 0.41 \pm 1.23$ & PLB 662, 102 (2008) & \pm 0.13 & \\
\hline$D^{0} \rightarrow K^{\mp} \pi^{ \pm} \pi^{0}$ & 281 & $-0.60 \pm 5.30$ & PRL 95, 231801 (2005) & \pm 0.40 & \\
\hline$D^{0} \rightarrow K^{\mp} \pi^{ \pm} \pi^{+} \pi^{-}$ & 281 & $+0.43 \pm 1.30$ & PRL 95, 231801 (2005) & \pm 0.33 & \\
\hline$D^{+} \rightarrow \pi^{0} \pi^{+}$ & 921 & $+2.31 \pm 1.24 \pm 0.23$ & hep-ex/1712.00619 & \pm 0.40 & \\
\hline$D^{+} \rightarrow \phi \pi^{+}$ & 955 & $+0.51 \pm 0.28 \pm 0.05$ & PRL 108, 071801 (2012) & \pm 0.04 & \\
\hline$D^{+} \rightarrow \eta \pi^{+}$ & 791 & $+1.74 \pm 1.13 \pm 0.19$ & PRL 107, 221801 (2011) & \pm 0.14 & \pm 0.01 \\
\hline$D^{+} \rightarrow \eta^{\prime} \pi^{+}$ & 791 & $-0.12 \pm 1.12 \pm 0.17$ & PRL 107, 221801 (2011) & \pm 0.14 & \\
\hline$D^{+} \rightarrow K_{S}^{0} \pi^{+}$ & 977 & $-0.363 \pm 0.094 \pm 0.067$ & PRL 109, 021601 (2012) & \pm 0.03 & \pm 0.03 \\
\hline$D^{+} \rightarrow K_{S}^{0} K^{+}$ & 977 & $-0.25 \pm 0.28 \pm 0.14$ & JHEP 02 (2013) 098 & \pm 0.05 & \\
\hline$D_{s}^{+} \rightarrow K_{S}^{0} \pi^{+}$ & 673 & $+5.45 \pm 2.50 \pm 0.33$ & PRL 104, 181602 (2010) & \pm 0.29 & \pm 0.03 \\
\hline$D_{s}^{+} \rightarrow K_{S}^{0} K^{+}$ & 673 & $+0.12 \pm 0.36 \pm 0.22$ & PRL 104, 181602 (2010) & \pm 0.05 & \\
\hline
\end{tabular}

SM prediction but could be enhanced by New Physics contribution, so there will be possible enhancement from new physics and Belle II expects a precision of $0.40 \%$. 

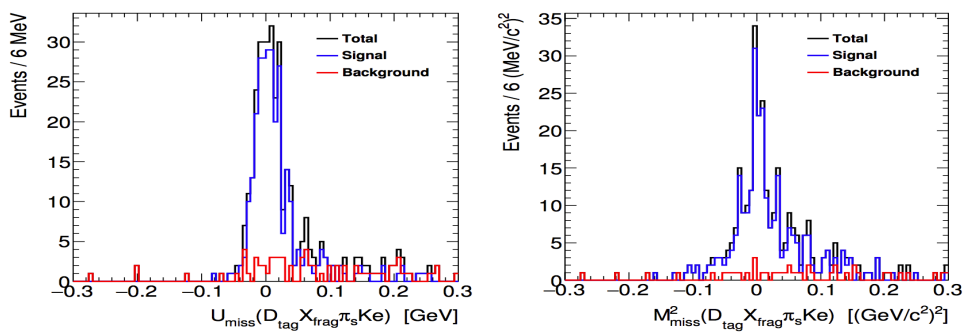

Fig. 2. The distribution of $U_{m i s s}$ and $M_{m i s s}^{2}$ where signal yields extracted from.

\section{Leptonic Decays}

The clean environment of a B-factory and the knowledge of the center-of-mass (CM) energy will allow Belle II to measure precisely the branching fractions of the leptonic decays $D_{(q)}^{-} \rightarrow \ell^{-} \bar{\nu}(q=d, s)$ and to extract the quantities $\left|V_{c q}\right| f_{D_{q}}$, where $f_{D_{q}}$ and $\left|V_{c q}\right|$ are decay constants and CKM matrix elements respondingly. By using the $f_{D_{q}}$ value computed with lattice QCD techniques, Belle II will significantly improve the Belle measurement of $\left|V_{c s}\right|$ and determine $\left|V_{c d}\right|$ with less than $2 \%$ uncertainty.

To study leptonic decays with missing energy from neutrino in $c \bar{c} \rightarrow$ $D_{\text {tag }}^{0+} D_{(q)}^{-} X_{\text {frag }}^{-0}$. Firstly, one need to reconstruct the "tag side" $D_{\text {tag }}$, like $D^{0}, D^{+}$ or $\Lambda_{c}^{+}$, nominally recoiling against the "signal side" $D_{(q)}^{-} \rightarrow(h) \ell^{-} \bar{\nu}(\ell=e / \mu)$. In addition, remaining pions, kaons and protons are grouped together into what is referred to "fragmentation side" $X_{\text {frag }}$. The missing momentum of neutrino $P_{\text {miss }}=P_{C M}-P_{D_{\text {tag }}}-P_{\text {frag }}-P_{\ell^{-}}\left(-P_{h}\right)$ is then constructed, and for signal processes $D_{(q)}^{-} \rightarrow(h) \ell^{-} \bar{\nu}$, the signal yields can be obtained by fitting the variable of neutrino $U_{m i s s}=E_{m i s s}-\left|\vec{P}_{m i s s}\right|$ which should peak at zero, instead of the missing mass $M_{\text {miss }}^{2}=P_{\text {miss }}^{2}$ for a larger resolution than $U_{\text {miss }}$ shown in Fig. 2. This method can also be applied to search for the invisible final state decays of $D^{0}$, like $D^{0} \rightarrow \nu \bar{\nu}$, which will be benefited from increased statistical sample.

\section{Flavour Tagging Technique: ROE Method}

Different from widespread $D^{0}$ flavor tagging method, named $D^{*}$ method here, which uses the charge of $\pi_{s}$ coming from $D^{*+} \rightarrow D^{0} \pi_{s}^{+}$or $D^{*-} \rightarrow \bar{D}^{0} \pi_{s}^{-}$to tag flavor, a new flavor tagging method, named ROE method, aims at increasing size of $D^{0}$ tagged candidates sample, and adding $D^{0}$ sample produced in $c \bar{c}$ events other than coming from $\mathrm{D}^{* \pm}$ decays.

The principle of the ROE method, illustrated in Fig. 3, uses the information of Rest Of Event. Since Cabbibo favored transition for a charm quark is $\bar{c} \rightarrow \bar{s}$, one strange meson in the ROE, namely a $\mathrm{K}^{+}(u \bar{s})$ or a $\mathrm{K}^{0}(\mathrm{~d} \bar{s})$, is produced. The ROE method is performed by selecting events with only one $K^{ \pm}$in the ROE, and using the charge of kaon to tag the $D^{0}$ flavor at the time of its production.

The amount of $D^{0}$ mesons tagged by ROE method is expected to be roughly same with that tagged by the charged $D^{*}$ method, which means that an additional 


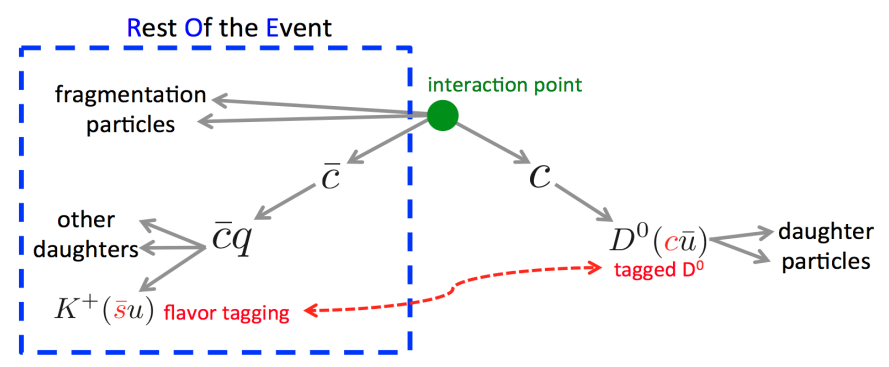

Fig. 3. The sketches of the ROE method. The events with only one $\mathrm{K}^{ \pm}$in the $\mathrm{ROE}$ are selected; the flavour of the neutral D meson is determined by the charge of the kaon.

$D^{0}$ sample with larger statistics is available for $D^{0}-\bar{D}^{0}$ mixing and $C P$ violation measurements. It is estimated that a $15 \%$ reduction of the statistics uncertainty on $A_{C P}\left(D^{0} \rightarrow K^{-} \pi^{+}\right)$can be achieved by combining $D^{*}$ tagging and ROE tagging method ${ }^{5}$.

\section{Conclusion}

Belle II at SuperKEKB will collect first data in 2018 and has a rich charm physics program. Considering impacts of the improved vertex resolution and particle indentification performances etc., with the targeted dataset of $50 \mathrm{ab}^{-1}$, Belle II is expected to obtain the $D^{0}-\bar{D}^{0}$ mixing and $C P$ violation parameters with about one magnitude improvement, more precise results on $C P$ asymmetries in $D$ decays, much improved leptonic decay measurements. A new $D$ flavor-tagging method, ROE method, will gain an additional $D^{0}$ sample as large as $D^{*}$ tag method to achieve more precise on $D^{0}-\bar{D}^{0}$ mixing and $C P$ violation measurements.

\section{Acknowledgments}

This work is supported in part by National Natural Science Foundation of China (NSFC) under contract Nos. 11475187 and 11521505; Key Research Program of Frontier Sciences, CAS, Grant No. QYZDJ-SSW-SLH011; and the CAS Center for Excellence in Particle Physics (CCEPP). We also thank Charm Work Group of the Belle II Collaboration for the studies in charm sector.

\section{References}

1. T. Abe et al. (Belle II Collaboration), Belle II Technical Design Report, KEKREPORT-2010-1, arXiv:1011.0352[physics.ins-det].

2. Belle II Collaboration \& B2TiP Theory Community, the Belle II Physics Book (to be published on PTEP).

3. L.-K. Li et al., Chin. Phys. C 41, 023001 (2017).

4. U. Nierste and A. Schacht, Phys. Rev. D 92, 054036 (2015).

5. G. De Pietro and G. Casarosa, BELLE2-NOTE-PH-2017-001 (2017). 